\title{
Checkpoints and restriction points in bacteria and eukaryotic cells
}

\author{
Stephen Cooper
}

\begin{abstract}
Summary
Bacterial checkpoints, analogous to those proposed to exist in eukaryotic cells, offer insights into the definition of a checkpoint. Examination of bacterial “checkpoint" or arrest phenomena illustrate problems with a too-casual application of the checkpoint idea to eukaryotic phenomena. The question raised here is whether there are cellular processes that "check" whether a cellular process is completed. It is possible that many eukaryotic "checkpoints" may not have "checking" functions. Some of the ubiquitous checkpoint phenomena widely described may be merely the result of the inherent incompleteness of earlier events preventing the initiation of subsequent events. BioEssays 28:1035-1039, 2006.

(C) 2006 Wiley Periodicals, Inc.
\end{abstract}

\section{Introduction}

It is arguable that the idea of checkpoint is one of the key elements of the current standard and dominant model of eukaryotic cell-cycle control. Checkpoints are postulated to be points within the eukaryotic cell cycle at which some cellular element "checks" that a particular cell function has been completed. If the processes or functions are incomplete, the postulated checkpoint element prevents subsequent processes from occurring. A simple hypothetical example would be a checkpoint where the cell checks that DNA synthesis is complete before allowing mitosis to start.

There are numerous definitions, in the literature, of the term "checkpoint". One early and key paper ${ }^{(1)}$ stated: "Control mechanisms enforcing dependency in the cell cycle are here called checkpoints. Elimination of checkpoints may result in cell death, infidelity in the distribution of chromosomes or other organelles, or increased susceptibility to environmental perturbations such as DNA damaging agents."

\footnotetext{
Department of Microbiology and Immunology, University of Michigan Medical School, Ann Arbor, Michigan 481090620.

E-mail: cooper@umich.edu

Funding agency: The National Science Foundation supported this work through Grant MCB-0323346.

DOI 10.1002/bies.20475

Published online in Wiley InterScience (www.interscience.wiley.com).
}

In a similar vein, the importance of checkpoints to normal passage of a cell through the cell cycle was cited: (2) "The cell cycle checkpoint mechanisms ensure the order of cell cycle events to preserve genomic integrity."

Another literature description of the importance of checkpoints noted:(3) "Cell cycle checkpoints are regulatory pathways that control the order and timing of cell cycle transitions and ensure that critical events such as DNA replication and chromosome segregation are completed with high fidelity."

A major text book ${ }^{(4)}$ has codified these ideas indicating the general description and purpose of checkpoints in regulating the cell cycle. Thus it is written: "... if some malfunction prevents the successful completion of [a cell cycle process] signals are sent to the control system to delay progress into the next phase. These delays provide time for the machinery to be repaired and also prevent the disaster that might result if the cell progressed prematurely to the next stage.

In most cells there are several points in the cell cycle, called checkpoints, at which the cycle can be arrested if previous events have not been completed. Entry into mitosis is prevented, for example, when DNA replication is not complete, and chromosome separation in mitosis is delayed if some chromosomes are not properly attached to the mitotic spindle."

The eukaryotic restriction point is widely regarded as a checkpoint that causes cells to arrest at a point in the $\mathrm{G}_{1}$-phase when growth conditions are limiting. ${ }^{(5)}$

It is proposed here that the concept of the eukaryotic cellcycle checkpoint has been overused, and needs a meticulous and rigorous re-examination. Upon rigorous analysis it may (and hopefully will) be seen that several of the phenomena regarded as signifying checkpoints involve internal control devices rather than external "checking" processes.

In order to explain why a reexamination of the eukaryotic checkpoint concept is needed, it will be instructive to look at some of the first checkpoint phenomena described. Checkpoint-like events were actually first described in bacteria though they were not called "checkpoints" when they were discovered, nor "restriction points" when they were proposed. But their similarities to several eukaryotic checkpoint phenomena are clear and their analysis brings out important concepts of relevance to eukaryotic cell biology. 


\section{Two bacterial checkpoints}

In 1961, Maaløe and Hanawalt ${ }^{(6)}$ studied the residual synthesis of DNA when bacterial cells were starved of an amino acid. When cells were starved of an amino acid, they found that rounds of DNA replication that were in progress were completed, but new rounds of replication were not initiated. They concluded that an amino acid had to be present, either made normally or supplied exogenously, in order to allow initiation of a new round of DNA replication.

In terms of checkpoints, we could say, in hindsight, that the replication of bacterial DNA is subject to an "amino acid checkpoint", where the cell "checks" that enough protein is made or all amino acids are present to allow initiation to occur. In terms of functional utility, we could propose that the cell "checks" that protein synthesis proceeds properly before allowing the initiation of DNA replication.

The bacterial "initiation checkpoint" described here is similar to the presumptive eukaryotic checkpoint for DNA synthesis control, whose onset requires some minimum amount of cell protein present so that the resulting daughter cells will have a minimum amount of protein that will enable the newborn cells to survive.

A second bacterial "checkpoint," described most clearly in 1968 by Helmstetter and Pierucci, ${ }^{(7)}$ is related to the completion of DNA replication in bacteria. When DNA synthesis is inhibited by UV irradiation, cell division is inhibited in most of the cells. Residual cell division occurs in cells later in the cell cycle, while cells earlier in the cell cycle do not divide. It was rigorously demonstrated that cell division is prevented in those cells with only one genome, while cells further along in the cell cycle - those with two complete genomes - can divide. In checkpoint terminology, there is a "DNA replication/ completion checkpoint" that checks whether DNA replication has been completed before cell division is allowed to proceed. In cells that have not completed DNA replication, cell division is inhibited. Analogous to eukaryotic cells where the failure to complete DNA replication forestalls mitosis, bacterial cells that fail to complete DNA replication stop binary fission, the bacterial equivalent of mitosis.

\section{What are checkpoints?}

Before analyzing these bacterial checkpoints in more detail, it is necessary to distinguish two different ideas regarding checkpoints. The main question is whether the checkpoint mechanism is "external" to the processes that are being checked. In a cell where DNA replication must be successfully completed before cell division proceeds, the question is whether there is an element or function that checks on the process of replication and its completion-only when the checking shows that the process has been completed is the signal given for mitosis. A true checkpoint must have two active domains, properties, or functions-one is to monitor the system being checked, and the other to emit a signal to prevent subsequent processes if the process that was checked was not completed. It is possible that the checking and controlling processes are part of a multi-element system, such that a checking element triggers other elements in a pathway to stop subsequent events from taking place. In either case, whether the checkpoint controls are within a single protein or composed of multiple proteins, there must be both checking and signaling elements.

In contrast to such external control points, which are outside of the system being checked, are internal control points, events within the system that must occur in order for subsequent processes to proceed. As we shall see, the two bacterial systems exemplify internal control points rather than "checkpoints."

Hartwell and Weinert ${ }^{(1)}$ in their original definition of the checkpoint concept were careful to make "externality" part of the definition of checkpoints. They noted that many events in the cell cycle occurred in an orderly and sequential manner. And further, there were dependencies where subsequent events required the completion of earlier events. Hartwell and Weinert clearly distinguish between the order determined by the properties of the elements themselves, and separate or "external" control elements that monitor the sequential completion processes. Their terminology serves to distinguish between a "substrate-product" order and a "specific control mechanism". These terms are analogous to the internal and external control points, as discussed here. Thus it is important to note that the original definition of checkpoints ${ }^{(1)}$ did distinguish between internal and external control systems, and the ideas presented here are related to whether there has been an indiscriminate application of the term "checkpoint" to different arrest phenomena.

Similar distinctions were emphasized by Nasmyth. ${ }^{(8)} \mathrm{He}$ wrote: "The key point is that cell cycle arrest caused by damage or incompletion of earlier cell cycle events can be caused not by damage or incompletion per se, but instead by specific surveillance mechanisms that detect mistakes and induce inhibitors of key cell cycle transitions....surveillance mechanisms that check completion have been likened to roadblocks where travelers are scrutinized and thus are often called checkpoints."

Nasmyth referred to the word "checkpoint" as a "shibboleth," indicating that this word has meaning to the cognoscenti but which may not be clearly understood by others and, in consequence, is often employed when it should not be. One problem with the application of the checkpoint idea is that the existence of a checkpoint is often inferred simply when a particular condition serves to align cells with a particular cellcycle configuration.

It may be helpful, at this point, to consider a simple analogy illustrating external and internal control points. Let us say that we are about to let one of our teenage children drive the family car for the first time. In order to ensure a modicum of safety, we

\section{BioEssays 28.10}


wish that he or she would drive less than 100 kilometers that evening. How can we ensure that this limit is enforced? One way is to install on the automobile a meter that continuously reads how many kilometers have been driven since the meter was reset. When the meter reaches 100 kilometers the meter, through its controlling functions, would shut off the engine. The meter is external to the engine, and has two functions. One function is to continuously read the status of the distance driven, and the second is to shut off the engine when a particular distance is reached. This is an analogue of an external control point.

An alternative system for controlling the distance driven is to merely put into the automobile enough liters of petrol sufficient to enable the car to drive 100 kilometers. (Presumably one would lock the tank so that refilling by your offspring would not be possible.) A check on the distance driven would not be based on some external checking system, but would be inherent in the nature of gasoline and an automobile engine. When the petrol was used up, the car would stop. This is an example of an internal control point.

Let us now examine the bacterial checkpoints described above in order to apply the ideas of external and internal control points.

\section{Understanding bacterial "checkpoints"}

It is currently understood that the process of initiation of bacterial DNA replication at a specific chromosomal origin is dependent on the accumulation of some amount of protein or cytoplasmic mass. DNA replication starts when protein has accumulated to a sufficient level to allow initiation to proceed. $^{(9)}$ Thus chloramphenicol, an inhibitor of bacterial protein synthesis, can inhibit initiation of DNA replication, yet allows rounds of replication in progress to continue. Most important, although the "protein accumulation checkpoint" acts at the initiation of DNA synthesis, the accumulation process occurs throughout the cell cycle and is cycleindependent. From this perspective, the idea of a "point" at which a control checks the cellular condition or acts is not clear.

An external control system would be a system in the cell that would continuously monitor whether or not a particular cell mass or cell protein content has been achieved. Although there are many possible results to be expected from the loss of an external control point that controlled initiation of DNA replication, one result might be the continuous initiation of DNA replication without the need for an accumulation of cell mass. Such a mutant has not been observed.

The accumulation of mass is presumably a surrogate for the continuous accumulation of some triggering protein (commonly believed to be $d n a A$ protein), until a sufficient amount has bound to the origin of replication. The accumulation of the triggering protein is the control system itself (i.e., an internal control system), with no other system (i.e. no external control system) monitoring whether enough protein has accumulated.

The bacterial system that prevents cell division (binary fission in the case of bacteria) from taking place unless DNA replication has been completed is an even more instructive example. Consider a culture of bacterial cells where the cells prior to the mid-point of the cell cycle have not completed DNA replication and do not have two separate chromosomes or nucleoids. Cells past the midpoint of the cell cycle will have two separate nucleoids because they have completed a round of DNA replication. When cells are irradiated with UV light, there will be cell division for one-half of a cell cycle. All cells past midpoint age, and thus having two nucleoids at the moment of irradiation, will divide. Cells prior to the midpoint at the time of irradiation, and thus with only one nucleoid, will not divide. The effect of UV irradiation is to damage DNA in such a way as to inhibit DNA replication. Thus, one could postulate a control system that "checks" that DNA is or is not damaged. This would be a UV-damage "checkpoint" in bacteria, and the checkpoint would be recognized by whether a cell with such damaged DNA could divide.

But, in the example presented here, all irradiated cells have damaged DNA, both those that do not divide (the younger cells), and those that do divide (the older cells). Checkpoint theory would postulate the existence of a system that would check on the damage to DNA and then signal cells with damaged DNA to eschew division. If such a system existed, all cells would have division inhibited.

Consider that cells with one genome have that genome in the center of the cell where the septum would normally be produced. The single genome or nucleoid in the center of the cell could, by its physical presence, prevent septum formation and thus inhibit binary fission or cell division. No "checking" system need be invoked. Inhibition of cell division is inherent in the fact that two separate genomes do not coexist in the cell. Cells with two genomes, even with damaged DNA, will now divide because the two genomes are at positions one-quarter and three-quarters of the cell length and the septum position at one-half of the cell length is not blocked. The results do not require invocation of a "checkpoint". There is only the failure to accomplish some process that is integral to the subsequent process being studied.

\section{Eukaryotic and bacterial "restriction points"}

It has been postulated that eukaryotic cells (mammalian cells in particular) have a particular point in the $\mathrm{G}_{1}$-phase at which cells make a decision whether to arrest at that point or to move forward to initiate DNA synthesis. Placement of cells in low serum arrests cells with a $\mathrm{G}_{1}$-phase amount of DNA. Cells prior to the "restriction point" when treated to suboptimal (e.g. low serum) growth media do not initiate DNA replication and are those that maintain $a G_{1}$-phase amount of DNA. Cells past 
the restriction point now initiate DNA replication, proceed to divide, and produce cells with a $\mathrm{G}_{1}$-phase amount of DNA. All cells are now "arrested in $\mathrm{G}_{1}$-phase" (more precisely, arrested "with a $\mathrm{G}_{1}$-phase amount of DNA"), thus defining a restriction/ decision point in the $\mathrm{G}_{1}$-phase of the cell cycle.

The restriction point can be considered a "checkpoint" in the same way that other checkpoints are defined. Here the definition would be that something in the cell checks whether a cell has accomplished some $\mathrm{G}_{1}$-phase-specific task before allowing DNA replication to proceed.

The arguments against the existence of the eukaryotic restriction point have been presented before ${ }^{(10-12)}$ and they need not be repeated here. Instead, it will be shown that, in bacteria, an analogous restriction point has been postulated (prior to the proposal of the eukaryotic restriction point) and our understanding of this bacterial control or restriction point supports earlier critiques of the restriction point proposal.

Prior to 1974 when Pardee postulated the existence of a restriction point in eukaryotic cells, ${ }^{(13)}$ Lark and Renger ${ }^{(14)}$ postulated the existence of two restriction points in bacteria. The two "restriction points" points were defined by their sensitivity or response to different concentrations of chloramphenicol. As it turned out, experimental and theoretical analysis of these bacterial restriction points indicated that their existence was the result of different rates of leakage of protein synthesis at different chloramphenicol concentrations. ${ }^{(15,16)}$

The experiments of Lark and Renger were quite simple. When two different concentrations of chloramphenicol were added to growing bacterial cells, the higher concentration of chloramphenicol allowed initiation only when added relatively close in time to initiation. In contrast, a low concentration of chloramphenicol allowed initiation even if added much earlier in time prior to initiation. That is, it appeared as though during the period of inhibition, the low concentration of chloramphenicol would allow more initiations of DNA replication to start than the high concentration. To be precise, when the point in the cell cycle at which the chloramphenicol was effective was analyzed, it was found that the high chloramphenicol point at which the cells escaped inhibition was later than the time when the low concentration restriction point existed. The high chloramphenicol inhibition point was closer to initiation than the low chloramphenicol inhibition point. In practical terms, one could imagine that high concentrations of chloramphenicol would effectively inhibit initiation if added more than, for example, 10 minutes prior to initiation. In contrast, the low concentration of chloramphenicol would have to be added earlier (perhaps 20-30 minutes) prior to initiation in order to restrict initiation. The low concentration of chloramphenicol defined an "early" restriction point compared to higher concentrations of chloramphenicol.

Simple experimental measurements of residual protein synthesis in the presence of different concentrations of chloramphenicol indicated that there was significant "leakage" of protein synthesis just at the concentrations studied. All of the results on the two bacterial restriction points could be accounted for by residual protein synthesis. ${ }^{(15,16)}$ Either there were an infinite number of restriction points, each defined by a different concentration of chloramphenicol, or there were no true restriction points and the finding of a point at which inhibition was effective if the cells were before that point and ineffective after that point could be simply accounted for by residual protein or mass accumulation.

Similar considerations have been applied to the eukaryotic restriction point. It has been shown that not only does the original evidence not support the existence of a restriction point, but further experiments attempting to understand the restriction point suggest that such a point does not exist. ${ }^{(10,12)}$

\section{The relationship between bacterial and eukaryotic phenomena}

It may be asked whether it is proper to use an analysis of bacteria to discuss the existence or non-existence of eukaryotic phenomena. It can be argued that just because some phenomenon does not exist in the "simpler" bacteria does not mean that the more complex "eukaryotes" may not have evolved such phenomena. This is a valid point. But that is not the purpose of the bacterial analogy. It is not argued that if something does not exist in bacteria it would not exist in eukaryotes. Rather the argument is more pedagogical than prescriptive. The bacterial systems allow a clear discussion of the phenomena that may be called checkpoints. For this reason, the bacterial systems highlight and illustrate problems with the widespread and possibly overused term "checkpoints" in eukaryotes.

To summarize the ideas presented here, it is proposed that much of the use of the term "checkpoint" applies to eukaryotic systems that do not clearly have checkpoint properties, where processes are "checked" before allowing subsequent processes to proceed. A rigorous application of checkpoint criteria should be used in order to prevent the casual overuse of the term "checkpoint".

\section{Acknowledgments}

Dr. Nanne Nanninga was extremely helpful with suggestions and moral support for this work.

\section{References}

1. Hartwell LH, Weinert TA. 1989. Checkpoints: controls that ensure the order of cell cycle events. Science 246:629-634.

2. Murakami $H$, Nurse P. 2000. DNA replication and damage checkpoints and meiotic cell cycle controls in the fission and budding yeasts. Biochem J 349:1-12.

3. Elledge SJ. 1996. Cell cycle checkpoints: preventing an identity crisis. Science 274:1664-1672

4. Alberts B, Johnson A, Lewis L, Raff M, Roberts K, et al. 2002. Molecular Biology of the Cell. New York City: Garland Science.

5. Planas-Silva MD, Weinberg RA. 1997. The restriction point and control of cell proliferation. Curr Opin Cell Biol 9:768-772

\section{BioEssays $\mathbf{2 8 . 1 0}$}


6. Maaløe O, Hanawalt P. 1961. Thymine deficiency and the normal DNA replication cycle. J Mol Biol 3:144-155.

7. Helmstetter CE, Pierucci O. 1968. Cell division during inhibition of deoxyribonucleic acid synthesis in Escherichia coli. J Bacteriol 95:16271633.

8. Nasmyth K. 1996. Viewpoint: putting the cell cycle in order. Science 274 1643-1645

9. Cooper S. 1997. Does the initiation mass for DNA replication in Escherichia coli vary with growth rate? Mol Microbiol 26:1138-1141.

10. Cooper S. 1998. On the proposal of a $G_{0}$ phase and the restriction point. FASEB J 12:367-373

11. Cooper S. 1981. The continuum model: application to $G_{1}$-arrest and $G_{0}$ In: Nicolini C, editor. Cell Growth. New York: Plenum Press. p 315-336.
12. Cooper S. 2003. Reappraisal of serum starvation, the restriction point, $\mathrm{G}_{0}$, and $\mathrm{G}_{1}$-phase arrest points. FASEB J 17:333-340.

13. Pardee AB. 1974. A restriction point for control of normal animal cell proliferation. Proc Natl Acad Sci USA 71:1286-1290.

14. Lark KG, Renger H. 1969. Initiation of DNA replication in Escherichia coli 15T-: chronological dissection of three physiological processes required for initiation. J Mol Biol 42:221-235.

15. Cooper S. 1974. A criterion for using chloramphenicol to define different processes in the initiation of $f$ synthesis in bacteria. J Theor Biol 46:117127.

16. Cooper S, Weusthoff G. 1971. Comment on the use of chloramphenicol to study the initiation of deoxyribonucleic acid synthesis. J Bacteriol 106:709-711.

BioEssays 28.10 1039 